\title{
Peptides \& enzymes
}

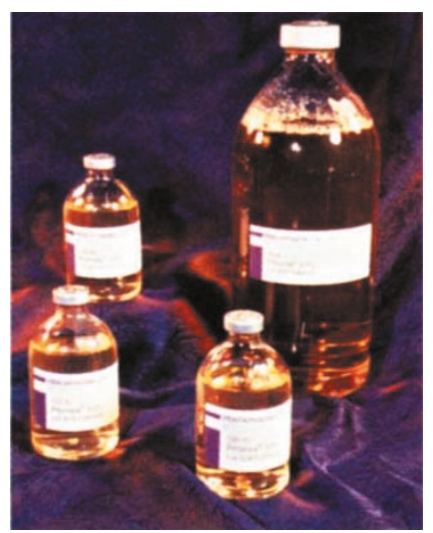

Pig preservative

Pentapharm (Basel, Switzerland) has developed an alternative to serum albumin for the stabilization of proteins. Prionex, a polypeptide fraction of porcine dermal collagen, maintains the biological activity of purified proteins during handling and storage. Prionex has advantages over serum albumin as it is filterable through $0.2 \mu \mathrm{m}$ membranes, is heat stable, free of bacteria, viruses, and endotoxins, and enhances storage times. It comes as a sterile, unpreserved aqueous solution containing $10 \%$ solids.

Tel: $+\mathbf{4 1 6 1 7 0 6 4 8 4 8}$

Fax: +41613199619

RSN: 1104

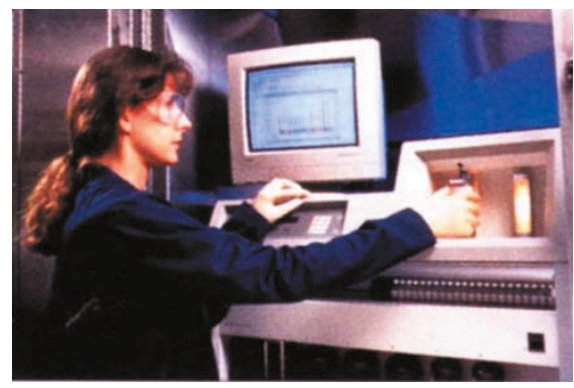

\section{Bioactive peptides}

Over 40 new bioactive peptides for use in signal transduction and basic peptide research are available from Sigma (St. Louis, MO), including CTOP, SPARC fragment 119-122, Orphanin FQ, Urocortin, Astressin, influenza hemaglutinin (HATag) peptide, and c-erb3 fragment 1265-1278. Sigma now offers over 600 bioactive peptides in 20 categories that meet stringent quality control and purity requirements, backed with comprehensive technical support.

Tel: +1 3147715765

Fax: +1 3147715757

http://www.sigma.sial.com

RSN: 1105

\section{DNA repair enzyme}

Trevigen's (Gaithersburg, MD) new DNA repair enzyme is specific for UV DNA lesions. A highly purified and stable recombinant form of the ultraviolet damage endonuclease (UVDE) from Schizosaccharomyces pombe, the enzyme efficiently excises UV-caused base modifications, including 6-4 photoproducts and cyclobutane pyrimidine dimers. It may be used with Trevigen's CometAssay to study UV damage at the single cell level.

Tel: +1800 TREVIGEN

Fax: +1301216 2801

http://www.trevigen.com

RSN: 1106

\section{Chromatography}

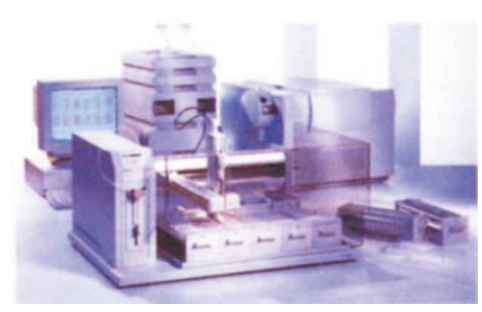

Microplate sampler

The HP 220 microplate sampler for injection and fraction collection from Hewlett Packard (Palo Alto, CA) makes high-throughput sample analysis easier by increasing sample throughput, shortening response times, and simplifying control. Combined with the HP 1100 series modules and systems for HPLC, it can store and handle more than 4,600 samples unattended. The sampler can be used in drug discovery, combinatorial chemistry, medicinal chemistry, and pharmacokinetic studies, and permits the use of various types and sizes of sample plates, vials, and test tubes.

Tel: +1 8002279770

http://www.hp.com

RSN: 1107

\section{Smaller is better}

A new chromatography column from TosoHaas (Stuttgart, Germany) has increased resolution and sensitivity for protein and peptide analysis. The TSKgel Super SW size exclusion column has a new $4 \mu \mathrm{m}$ silica particle size, which has shown a $30 \%$ improvement in sample resolution and a $50 \%$ reduction in analysis time. The column's increased sensitivity is useful for researchers with limited sample quantities, as the narrow bore diameter means less mobile phase consumption and less sample volume.

Tel: +49711 132570

Fax: +49711 1325789

RSN: 1108

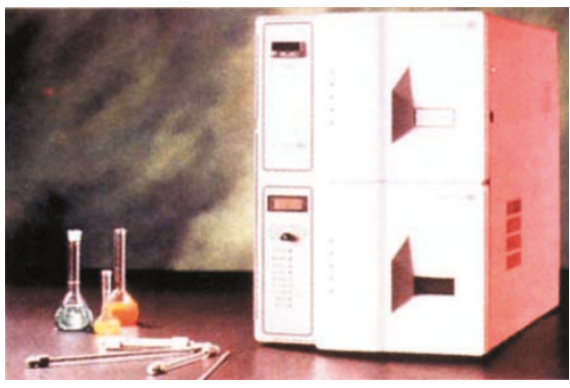

\section{Nitrogen detection}

A new HPLC detector from ChemLab Scientific Products (Hornchurch, UK) is sensitive to nitrogen, overcoming obstacles associated with traditional HPLC detection. The Antek 8060 is able to quantify an unknown without using the unknown as a standard because it gives an equimolar response to nitrogen only. This eliminates the need for derivatization and allows quantitation of all nitrogen-containing compounds. The Antek 8060 can quantify product yields, enhance structure-function and synthetic peptide studies, and can also be used in DNA mapping.

Tel: +441708476162

Fax: +44 1708707778

101447.704@compuserve.com

RSN: 1109

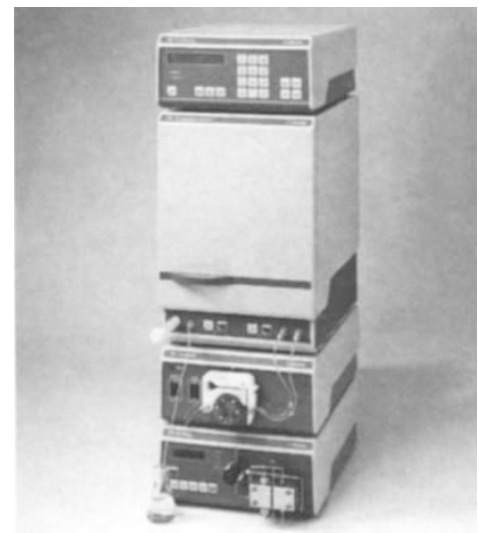

\section{Instrumentation}

The Metrohm (Herisau, Switzerland) IC System gives users the freedom to perform suppressed or nonsuppressed ion chromatography. Separate modules are available that allow units to be retrofitted to existing IC/HPLC systems, and the unique multichamber design of the suppressor module eliminates blockage, leaking, and back pressure. The injection valve and pump run without pressurized gas, eliminating additional maintenance.

Tel: +41 713538521

Fax: +41713538901

RSN: 1110 